\title{
Effect of Ta content on young's modulus and microstructures of binary Ti-Ta alloys
}

\author{
Y. L. Zhou ${ }^{1}$, M. Niinomi ${ }^{2}$, T. Akahori ${ }^{2}$ and Gunawarman ${ }^{2}$ \\ ${ }^{1}$ Department of Production Systems Engineering, Graduate Student, Toyohashi University of Technology, \\ Toyohashi 441-8580, Japan \\ 2 Department of Production Systems Engineering, Toyohashi University of Technology, Toyohashi \\ 441-8580, Japan
}

\begin{abstract}
The effect of Ta content on Young's modulus and microstructures of binary Ti-Ta alloys with different mass percents of Ta from $10 \%$ to $80 \%$ was investigated in order to find a Ta content that gives the suitable Young's modulus for biomedical applications. The experimental results show that both the crystal structure and Ta content have the strong effect on Young's modulus of binary Ti-Ta alloys. The $\alpha$ " Ti-Ta alloy with mass percents of $30 \%$ Ta and metastable $\beta$ Ti-Ta alloy with $70 \%$ Ta have the nearly same lowest Young's modulus under the given solution treatment. The microstructures of Ti-Ta quenched alloys are very sensitive to Ta content. The quenched alloys exhibit the plate-like HCP martensite $\left(\alpha^{\prime}\right)$ structure at a Ta content between 0 and 20 mass $\%$, the acicular orthorhombic martensite $(\alpha$ ") structure at a Ta content between 30 and 50 mass $\%$, metastable $\beta+\alpha$ " structure at a Ta content of 60 mass $\%$, and only metastable $\beta$ structure at a Ta content more than 60 mass $\%$.
\end{abstract}

Key words: Young's modulus, Microstructure, Ti-Ta alloys, Solution treatment, Biomedical materials

\section{INTRODUCTION}

$\beta$ Ti alloys or metastable $\beta$ Ti alloys, composed of non-toxic element $\mathrm{Nb}$, Ta, Mo and $\mathrm{Zr}$ have become one of the most attractive biomedical implant materials because of their superior bio-compatibility, excellent corrosion resistance, and better formability. However, the current bio-Ti alloys, which still possess much higher elastic modulus as compared with that of a human bone (max. $30 \mathrm{GPa}$ ), can lead to premature failure of the implant [1]. Therefore it is necessary to further reduce Young's modulus of Ti alloys for biomedical applications. Song et al. [1] have calculated the binding energies between titanium and various alloying elements within the cluster by using the discrete variation cluster method, from which strength and modulus are then estimated. They have suggested that $\mathrm{Ta}, \mathrm{Nb}, \mathrm{Hf}, \mathrm{Zr}$ and $\mathrm{Mo}$ are suitable alloying elements in enhancing the strength and reducing the modulus of the $\beta$ type Ti alloys. Up to now, a little research work has been done about binary Ti-Ta alloys.

In this study, the effect of Ta content on Young's modulus and microstructures of binary Ti-Ta alloys was investigated in order to find a binary $\mathrm{Ti}-\mathrm{Ta}$ alloy that gives the suitable Young's modulus for biomedical applications, and to provide a useful data for developing new $\mathrm{Ti}$ alloys with much lower Young's modulus for biomedical applications.

\section{EXPERIMENTAL PROCEDURE}

Corresponding author:

Y. L. Zhou, shuu@sp-mac4.tutpse.tut.ac.jp

\subsection{Fabrication of binary $\mathbf{T i}$-Ta alloys}

The binary Ti-Ta alloys with Ta contents from 10 to 80 mass $\%$ were prepared from high purity sponge titanium and sheet $\mathrm{Ta}$ in the appropriate proportion. Before melting, the constituents were cleaned in methanol to remove possible surface contaminants. Melting was carried out in water-cooled copper hearth non-consumable tungsten electrode tri-arc furnace containing a high purity argon atmosphere. Prior to melting, a definite amount of oxygen getter was melted in the furnace which had been evacuated and flushed five times with purified argon. Owing to the big difference in melting point (Ti: $1953 \mathrm{~K}$, Ta: $3269 \mathrm{~K}$ ) and density (Ti: $4.5 \mathrm{~g} / \mathrm{cm}^{3}$, Ta: $16.6 \mathrm{~g} / \mathrm{cm}^{3}$ ) between the two elements, the designed alloys were melted more than ten times, and each time were held in the molted state for 3-4 minutes. The oval ingots were flipped between each melting to promote chemical homogeneity.

All ingots were homogenized at $1273 \mathrm{~K}$ for $21.6 \mathrm{ks}$ to remove any residual microscopic segregation in vacuum, and then rolled to the plates of $3 \mathrm{~mm}$ thick by total reduction of $80 \%$. The rolled plates were subjected to the solution treatment at $1223 \mathrm{~K}$, which is above $\beta$ transus temperature, for $3.6 \mathrm{ks}$ followed by rapid quenching in ice water.

The compositions of the designed alloys were checked by comparing the weights of the initial materials with those of the ingots. The weight loss rates were found between $0.3 \sim 0.65 \%$. At the same time, the densities of the designed alloys were measured by the experiment, the density error rates were found between $0.29 \sim 0.68 \%$. 
These indicate that the actual composition of each designed alloy is very close to its nominal composition.

\subsection{Microstructures}

The microstructures were investigated by a light microscopy and scanning electron microscopy (SEM). The phase constitution was detected using an $\mathrm{X}$-ray diffraction analysis operated at $40 \mathrm{kV}$ and $30 \mathrm{~mA}$. The samples were prepared by grinding and polishing followed by etching in $10 \mathrm{vol} \% \mathrm{HF}, 15 \mathrm{vol} \% \mathrm{HNO}_{3}$ and 75 vol\% $\mathrm{H}_{2} \mathrm{O}$.

\section{3. Young's modulus and damping measurement}

After the solution treatment, the rectangular bar specimens with a cross-section of $10 \mathrm{~mm} \times 2 \mathrm{~mm}$ and 55 $\mathrm{mm}$ length were machined from the cold-rolled plates along longitudinal direction. These specimens were then grounded and polished, and then used to determine the dynamic Young's modulus and damping by the resonance vibration method at room temperature. The specimen was supported horizontally at its nodal points by two pairs of fine nickel wires. The sample was driven electrostatically in flexural vibration. The vibration amplitude was recorded as a function of frequency. The internal friction, $Q^{-1}$, was calculated from the resonance curve according to [2]

$Q^{-1}=\left(\log \frac{D_{1}}{D_{2}}+\log \frac{D_{2}}{D_{3}}+\cdots+\log \frac{D_{n-1}}{D_{n}}\right)^{-1}$

where $D_{1}$ is the maximum amplitude at a resonance frequency $f_{\mathrm{r}}$, and $D_{\mathrm{n}}$ is the $n$th amplitude of resonance curve. The dynamic Young's modulus, $E$, was calculated according to [2]

$$
E=0.9694 m L^{3} f_{r}^{2} /\left(w d^{3}\right)
$$

where $m, L, \mathrm{w}$ and $d$ are the mass, the length, width and the thickness of specimens, respectively. For each kind of designed Ti-Ta alloys, more than 3 pieces specimens were used for the measurement of dynamic Young's modulus and damping in order to minimize the experimental error caused by measurement and the possible chemical inhomogeneity

\subsection{Hardness measurement}

The hardness measurement was carried out using a micro-hardness instrument with a load of $98 \mathrm{~N}$ for $15 \mathrm{sec}$. The samples for hardness measurement were grounded and polished.

\section{RESULTS AND DISCUSSION}

\subsection{XRD results}

XRD analysis results in Fig. 1 show that the Ti-Ta quenched alloys have the martensite $\left(\alpha^{\prime}\right)$ structure at Ta contents between 0 and 20 mass $\%$, and only orthorhombic martensite ( $\alpha$ ") phase at Ta contents from 30 to 50 mass $\%$. While at a Ta content of 60 mass $\%$ the alloy consists of the metastable $\beta$ and $\alpha$ " phase, and the alloys comprise entirely of metastable $\beta$ phase when Ta content is more than 60 mass\%. This result is consistent with the early result of Fedotov et al [3].

Bagariatskii et al. [4] found that the increase in alloying content in a number of binary quenched $\mathrm{Ti}$ alloys (Ti-Mo, Ti-V, Ti-Nb and etc.) could cause the normally observed hexagonal $\alpha$ ' structure to be distorted and transformed into an orthorhombic $\alpha$ " phase. Fedotov et al. [3] reported that, in Ti-Ta system, the martensitic structure changes from hexagonal $\left(\alpha^{\prime}\right)$ to orthorhombic $(\alpha ")$ at around 26 mass\% Ta. However, Bywater et al [5] found that the martensitic structure changes from hexagonal $\left(\alpha^{\prime}\right)$ to orthorhombic ( $\left.\alpha^{\prime \prime}\right)$ at around 22 mass\% Ta. The experimental discrepancy is possibly caused by the effect of different level of impurities, microscopic chemical inhomogeneity and very near positions of some XRD peaks between $\alpha$ ' and $\alpha$ " phase. The result of

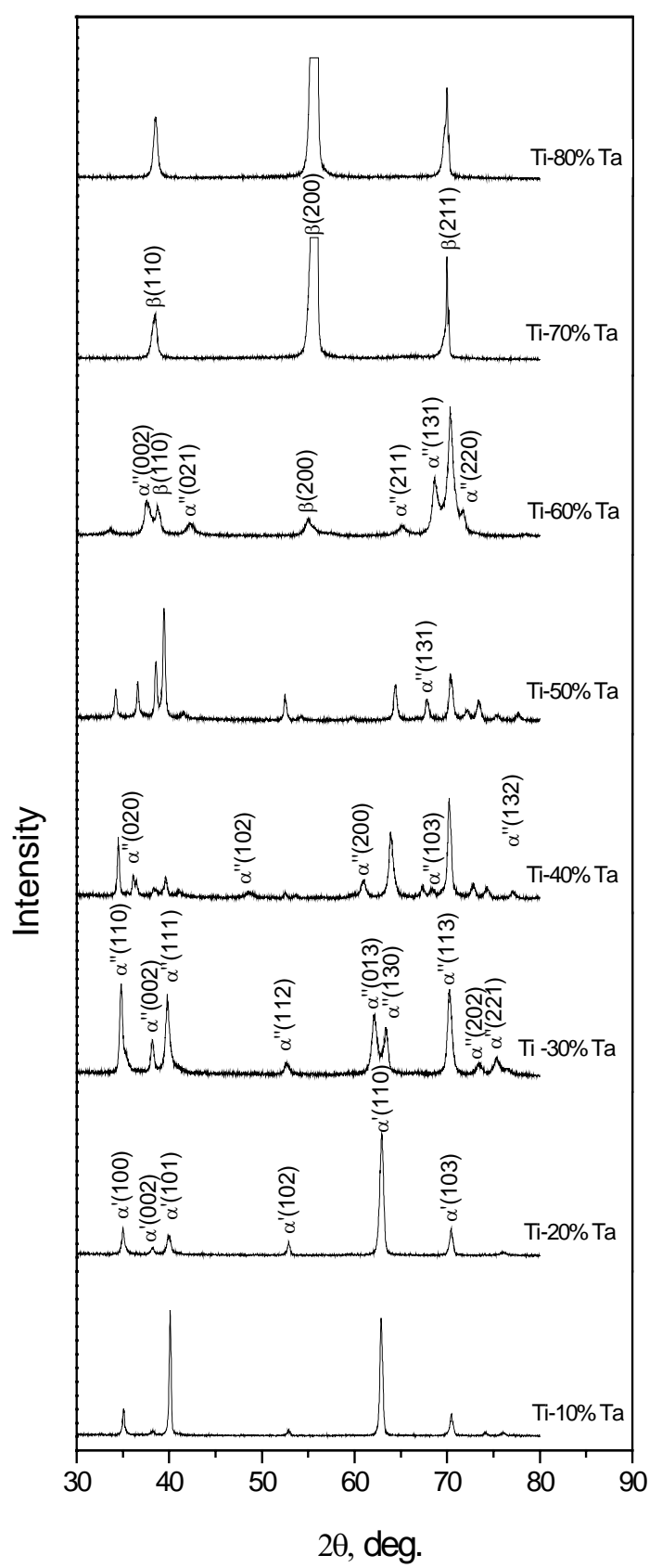

Fig. 1. XRD patterns of Ti-Ta alloys 

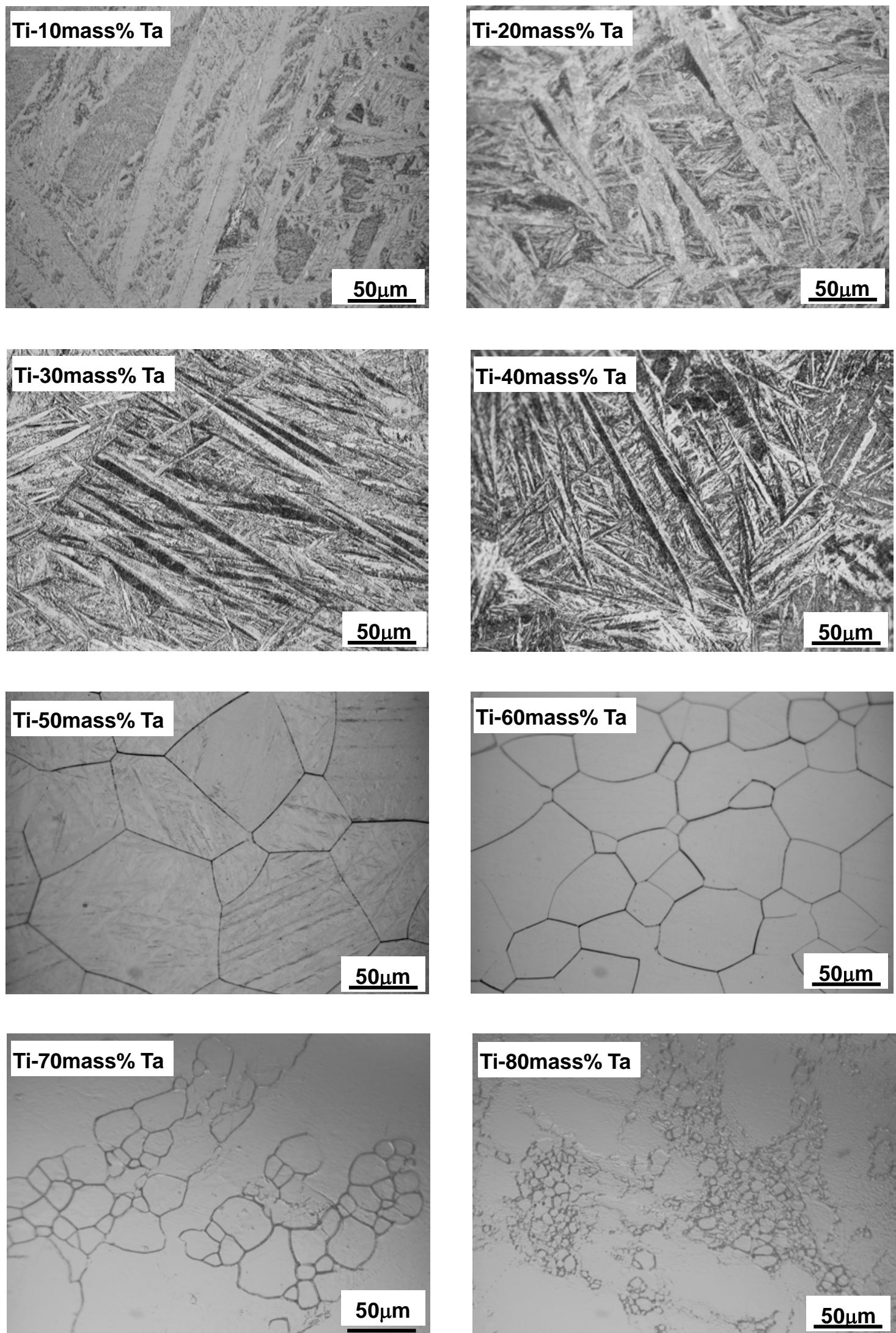

Fig. 2. Light micrograph of Ti-Ta alloys 
Fedotov et al. [3] is supported by the present study where the alloy with 25 mass\% Ta composed of both $\alpha^{\prime}$ and $\alpha$ "' phases.

\subsection{Microstructures}

The microstructures of the designed Ti-Ta alloys are shown in Fig. 2. Combining with the XRD results, Ti-Ta alloys exhibit the plate-like martensite $\alpha$ ' at Ta contents 10 mass $\%$ and 20 mass $\%$, the acicular martensite $\alpha "$ at Ta contents from 30 mass $\%$ to 50 mass $\%$. When the Ta content is increased to 60 mass $\%$, a significant amount of equiaxed retained $\beta$ phase is observed. The $\alpha$ " phase is not found because of the limited resolution of light microscopy. However, it can be observed by SEM as shown in Fig. 3. Only $\beta$ phase is observed when the Ta content is more than 60 mass\%.

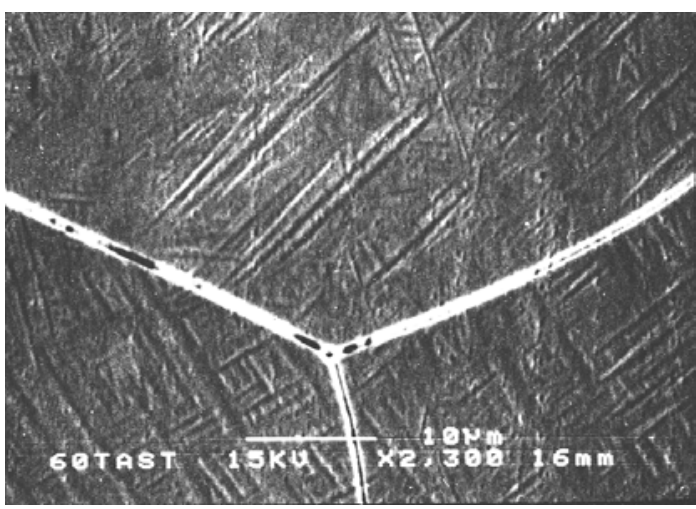

Fig. 3. SEM micrograph of Ti-60 mass $\%$ Ta

\subsection{Effect of Ta content on Young's modulus and damping of binary $\mathrm{Ti}-\mathrm{Ta}$ alloys}

Figure 4 shows that the dynamic Young's modulus of binary $\mathrm{Ti}-\mathrm{Ta}$ quenched alloys greatly depends on both phase structure and Ta content. The dynamic Young's modulus firstly decreases linearly with the increase of Ta content, and reaches to the minimum value at 30 mass $\%$ Ta. It gradually increases up to 50 mass $\% \mathrm{Ta}$, and then drops again to reach to the minimum value at 70 mass\% Ta. After that, the further increase in Ta content naturally leads to an increase in Young's modulus until near to that of pure Ta. This trend is very similar to the other $\beta$ isomorphic system ( $\mathrm{Ti}-\mathrm{Nb}$, Ti-Mo and etc.).

The changes of this nature in the elastic properties of the alloys are due to alteration in their structures and compositions (Ta content). Referring to the XRD result, it can be found that, the dynamic Young's modulus keeps on to decrease with the increase of Ta content from 10 to 20 mass\% despite of their same crystal structures in hexagonal martensite $\alpha$ '. On the contrary, the dynamic Young's modulus keeps on to increase in the alloying with Ta from 30 to 50 mass \% and from 70 to 80 mass \% although their crystal structures are the same martensite $\alpha$ " and metastable $\beta$, respectively. It is well known that, Young's modulus, one of intrinsic nature of materials, is determined by the bonding forces between atoms, and can be slightly affected by alloying addition, heat treatment and plastic deformation [6,7]. Since Young's modulus is not sensitive to the grain size and morphology of materials [7,8], and the same heat treatment and plastic deformation were carried out in the present study. Those indicate that $\mathrm{Ta}$ content has the strong effect on Young's modulus of Ti-Ta alloys, and the effect of composition is different for the different crystal structure.

It is generally accepted that only two stable phases namely $\alpha$ (hcp) and $\beta$ (bcc) and three metastable phases namely martensite $\alpha^{\prime}, \alpha^{\prime}$ and $\omega$ (hcp) exist in Ti alloys. The $\beta$ phase has the lowest Young's modulus; $\omega$ phase has the highest among those phases [9]. This is supported by the results of the present study where the alloys containing respectively $\alpha$ " and $\beta$ phase have the minimum elastic modulus. From the point of view of Young's modulus, the alloy with about 30 mass $\%$ Ta that contains $\alpha$ " phase and the alloy with about 70 mass $\% \mathrm{Ta}$ that contains metastable $\beta$ phase have great potential to be the new candidate alloys for biomedical applications.

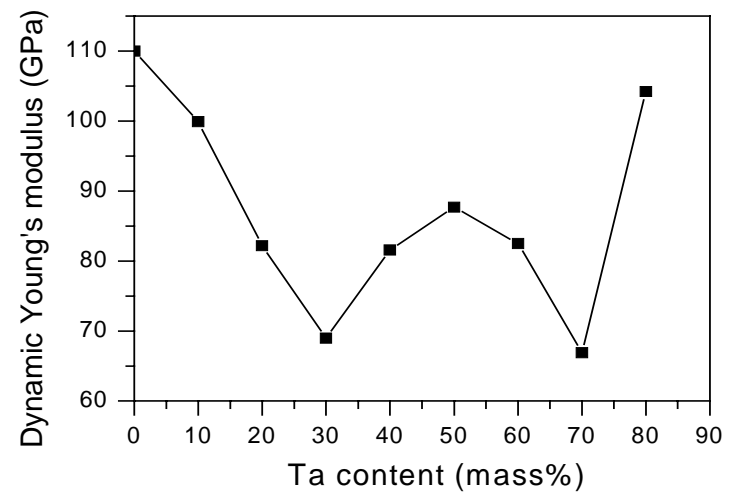

Fig. 4. Young's modulus and Ta content

The previous investigations $[10,11]$ found that, the damping capacity is sensitive to phase structures, and the damping peaks are often correlated with the phase change. The observed higher damping values at room temperature are found due to the presence of martensite $\alpha$ " or metastable $\beta$. The results in the present study agree with these reports. As shown in Fig. 5, there are two damping peaks, one is at 30 mass $\%$ Ta and the other one is at 60 mass\% Ta. This is in good agreement with the XRD results where one peak corresponds to transformation of $\alpha$ ' to $\alpha$ " phase, the other corresponds to the appearance of metastable $\beta$ phase.

\subsection{Hardness}

Hardness is one of the important properties that determine suitability of materials for biomedical applications. The hardness values of Ti-Ta quenched alloys, an average of twenty measuring data, vary from 175 to $218 \mathrm{VHN}$, and critically depend on Ta content under the given solution treatment. As shown in Fig. 6, the hardness values of all Ti-Ta alloys are higher than that of pure Ti where 50 mass $\%$ Ta alloy with $\alpha$ " phase has the lowest hardness. While the hardness is maximized at 60 mass $\%$ Ta alloy with $\alpha$ " and $\beta$ phases, 
which is hardened by both the solid solution effect and precipitation strengthening.

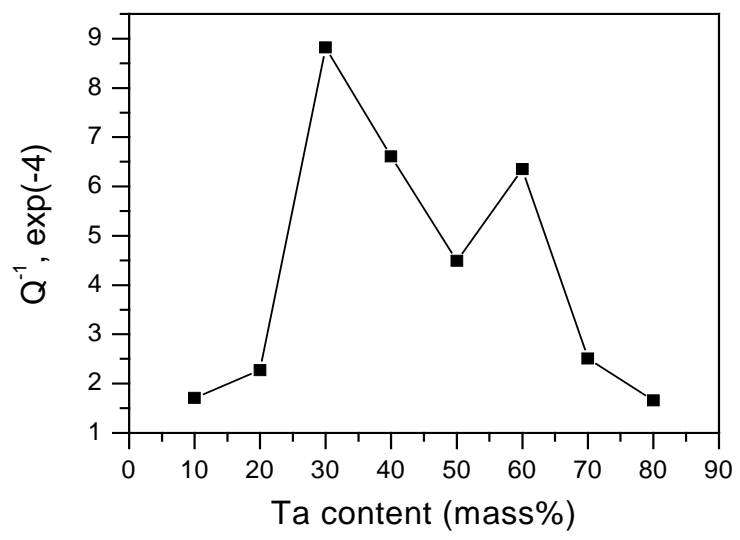

Fig. 5. Variation of damping and Ta content

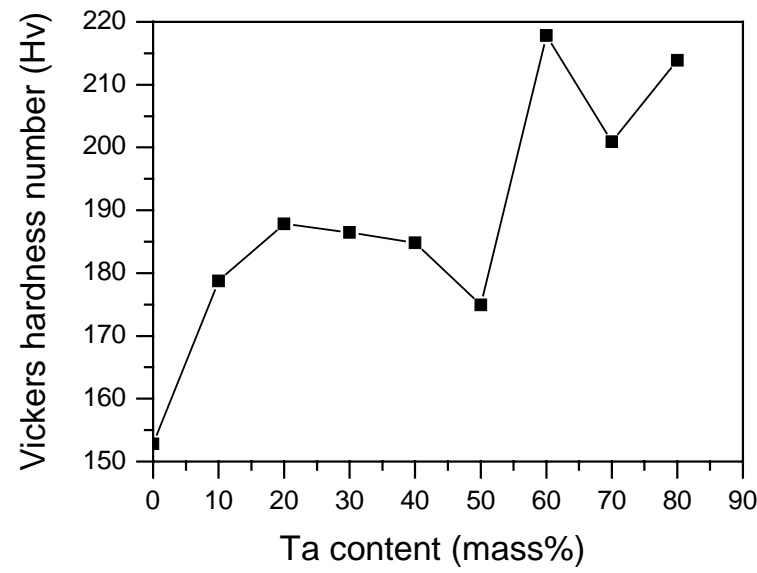

Fig. 6. Vickers hardness and Ta content

\section{CONCLUSIONS}

The effect of Ta content on Young's modulus and microstructures of binary Ti-Ta alloys was investigated in the present study. The microstructures of the binary Ti-Ta alloys were investigated using a light microscope, an SEM and $\mathrm{X}$-ray diffraction analysis. The dynamic Young's modulus was measured by the resonance vibration method at room temperature. The results can be summarized as follows:

(1) Both the crystal structures and Ta content have the strong effects on Young's modulus of binary Ti-Ta alloys.

(2) Ti-Ta binary alloy Ta-30 mass $\%$ Ta with $\alpha$ " martensite phase and Ti-70 mass \% Ta with metastable $\beta$ phase with have the nearly same lowest Young's modulus under the given solution treatment. Thus, these alloys have great potential to be the new candidate alloys for biomedical applications from the point of view of Young's modulus.

(3) Microstructures of binary Ti-Ta alloys are very sensitive to Ta content. The quenched alloys exhibit the martensite $\left(\alpha^{\prime}\right)$ structure at a Ta content below 20 mass $\%$, the orthorhombic martensite $(\alpha ")$ structure at a Ta content from 30 to 50 mass $\%$, metastable $\beta+\alpha$ " structure at a Ta content of 60 mass $\%$, and metastable $\beta$ structure at a Ta content more than 60 mass $\%$.

(4) Among all Ti-Ta alloys, 50 mass $\%$ Ta alloy with $\alpha$ " phase has the lowest hardness, while the $\beta+\alpha$ " phase 60 mass \% Ta alloy has the highest hardness.

\section{REFERENCES}

1. Y. Song, D. S. Xu, R. Yang, D. Li, W. T. Wu, and Z.X. Guo. Theoretical study of the effects of alloying elements on the strength and modulus of $\beta$-type bio-titanium alloys. Mater. Sci. and Eng. A 260, 1999, pp. 269-274.

2. Introduction of Young's modulus measure system (JE-RT 3). Technical Manual, Nippon Techno-Plus Co. Ltd., Japan. (In Japanese)

3. S. G. Fedotov, T. V. Chelidez, Yu. K. Kovneristyy and V. V. Sanadze. Phase transformation during hearting of metastable alloys of the Ti-Ta system. Phys. Met. Metall. vol. 62, No. 2, pp.109-113, 1986.

4. Iu. A. Bagariaskii, G. I. Nosova, and T. V. Tagunova. Factors in the formation of metastable phases in titanium-base alloys. Technical Physics, 1959,3, pp.1014-1018.

5. K. A. Bywater and J. W. Christian. Martensitic transformations in titanium-tantalum alloys, Phil. Mag. A, 1972, vol. 25, pp. 1249-1274.

6. George E. Dieter. Mechanical metallurgy. McGRAW-Hill KOGAKUSHA, LTD, Second Edition.

7. Z. X. Cui. Metallography and heat treatments. Mechanical Industry Press, 2000. (In Chinese)

8. Y. L. Hao, M. Niinomi, D. Kuroda, K. Fukunaga, Y. L. Zhou, R. Yang and A. Suzuki. Young's modulus and mechanical properties of Ti-29Nb-13Ta-4.6Zr in relation to $\alpha$ " martensite. Metall. and Mater. Trans. A, Vol. 33, 2002. pp. 3137-3144.

9. E. W. Collings. The physical metallurgy of titanium alloys. ASM, Metals Park, OH, 1984.

10. Y. T. Lee and G. Welsch. Young's modulus and damping of Ti-6Al-4V alloy as a function of heat treatment and oxygen concentration. Mater. Sci. and Eng. A 128, 1990, pp.77-89.

11. Y. T. Lee and G. Welsch. Titanium' 80 Science Technology, Proc. 5th Int. Conf. On Titanium, 1984, pp.1689-1696. 